\title{
valr: Reproducible genome interval analysis in $\mathbf{R}$ [version 1; peer review: 2 approved]
}

\author{
Kent A. Riemondy (iD1, Ryan M. Sheridan², Austin Gillen1, Yinni Yu², \\ Christopher G. Bennett ${ }^{3}$, Jay R. Hesselberth (D) 1,2
}

${ }^{1}$ RNA Bioscience Initiative, University of Colorado School of Medicine, Aurora, CO, 80045, USA

2Department of Biochemistry and Molecular Genetics, University of Colorado School of Medicine, Aurora, CO, 80045, USA

${ }^{3}$ ComAnalyzeIT LLC, Fort Collins, CO, 80525, USA

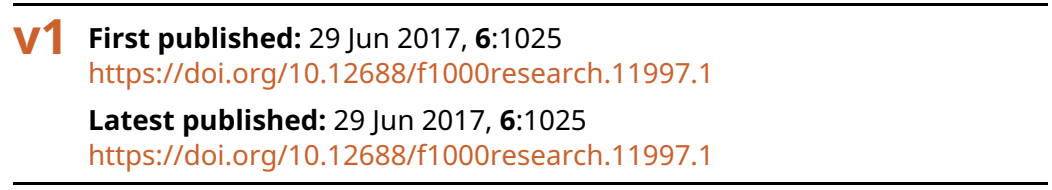

\section{Abstract}

New tools for reproducible exploratory data analysis of large datasets are important to address the rising size and complexity of genomic data. We developed the valr $\mathrm{R}$ package to enable flexible and efficient genomic interval analysis. valr leverages new tools available in the "tidyverse", including dplyr. Benchmarks of valr show it performs similar to BEDtools and can be used for interactive analyses and incorporated into existing analysis pipelines.

\section{Keywords}

Genomics, Intervals, BEDtools, reproducibility, R, RStudio

This article is included in the RPackage

gateway.

\section{Open Peer Review}

Approval Status

1

2

version 1

29 Jun 2017

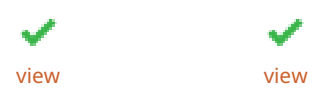

1. Robert A. Amezquita, Yale University, New Haven, USA

2. Ryan K. Dale iD, National Institutes of Health, Bethesda, USA

Any reports and responses or comments on the article can be found at the end of the article. 
Corresponding author: Jay R. Hesselberth (jay.hesselberth@gmail.com)

Author roles: Riemondy KA: Conceptualization, Software, Writing - Review \& Editing; Sheridan RM: Conceptualization, Software, Writing - Review \& Editing; Gillen A: Software; Yu Y: Software, Writing - Review \& Editing; Bennett CG: Software, Writing - Review \& Editing; Hesselberth JR: Software, Writing - Original Draft Preparation

Competing interests: No competing interests were disclosed.

Grant information: This work was supported by the RNA Bioscience Initiative (funded by a Transformational Research Award from the University of Colorado School of Medicine), a grant from the National Institutes of Health (R35 GM119550 to J.H.), the Colorado Office of Economic Development and International Trade (CTGGI 2016- 2096), the BioFrontiers Computing Core at the BioFrontiers Institute, University of Colorado at Boulder and the Intramural Research Program of the National Library of Medicine.

The funders had no role in study design, data collection and analysis, decision to publish, or preparation of the manuscript.

Copyright: (c) 2017 Riemondy KA et al. This is an open access article distributed under the terms of the Creative Commons Attribution License, which permits unrestricted use, distribution, and reproduction in any medium, provided the original work is properly cited.

How to cite this article: Riemondy KA, Sheridan RM, Gillen A et al. valr: Reproducible genome interval analysis in R [version 1; peer review: 2 approved] F1000Research 2017, 6:1025 https://doi.org/10.12688/f1000research.11997.1

First published: 29 Jun 2017, 6:1025 https://doi.org/10.12688/f1000research.11997.1 


\section{Introduction}

A routine bioinformatic task is the analysis of the relationships between sets of genomic intervals, including the identification of DNA variants within protein coding regions, annotation of regions enriched for nucleic acid binding proteins, and computation of read density within a set of exons. Command-line tools for interval analysis such as BEDtools $S^{1}$ and BEDOPS ${ }^{2}$ enable analyses of genome-wide datasets and are key components of analysis pipelines. Analyses with these tools commonly combine processing intervals on the command-line with visualization and statistical analysis in R. However, the need to master both the command-line and R hinders exploratory data analysis, and the development of reproducible research workflows built in the RMarkdown framework.

Existing R packages developed for interval analysis include IRanges ${ }^{3}$, bedr ${ }^{4}$, and GenometriCorr ${ }^{5}$. IRanges is a Bioconductor package that provides interval classes and methods to perform interval arithmetic, and is used by many Bioconductor packages. bedr is a CRAN-distributed package that provides wrapper R functions to call the BEDtools, BEDOPS, and tabix command-line utilities, providing out-of-memory support for interval analysis. Finally, GenometriCorr provides a set of statistical tests to determine the relationships between interval sets using IRanges data structures. These packages provide functionality for processing and statistical inference of interval data, however they require a detailed understanding of S4 classes (IRanges) or the installation of external command-line dependencies (bedr). Additionally, these packages do not easily integrate with the recent advances provided by the popular tidyverse suite of data processing and visualization tools (e.g. dplyr, purrr, broom and ggplot 2$)^{6}$. We therefore sought to develop a flexible $\mathrm{R}$ package for genomic interval arithmetic built to incorporate new $\mathrm{R}$ programming, visualization, and interactivity features.

\section{Methods}

Implementation

valr is an $\mathrm{R}$ package that makes extensive use of dplyr, a flexible and high-performance framework for data manipulation in $\mathrm{R}^{7}$. Additionally, compute intensive functions in valr are written in $\mathrm{C}++$ using Rcpp to enable fluid interactive analysis of large datasets ${ }^{8}$. Interval intersections and related operations use an interval tree algorithm to efficiently search for overlapping intervals ${ }^{9}$. BED files are imported and handled in R as data_frame objects, requiring minimal pre or post-processing to integrate with additional $\mathrm{R}$ packages or command-line tools.

\section{Operation}

valr is distributed as part of the CRAN R package repository and is compatible with Mac OS X, Windows, and major Linux operating systems. Package dependencies and system requirements are documented in the valr CRAN repository.

\section{Use cases}

To demonstrate the functionality and utility of valr, we present a basic tutorial for using valr and additional common use cases for genomic interval analysis.

\section{Basic usage}

Input data. valr provides a set of functions to read BED, BEDgraph, and VCF formats into R as convenient tibble (tbl) data_frame objects. All tbls have chrom, start, and end columns, and tbls from multi-column formats have additional pre-determined column names. Standards methods for importing data (e.g. read.table, readr: :read_tsv) are also supported provided the constructed dataframes contain the requisite column names (chrom, start, end). Additionally, valr supports connections to remote databases to access the UCSC and Ensembl databases via the $\mathrm{db}$ ucsc and $\mathrm{db}$ ensembl functions.

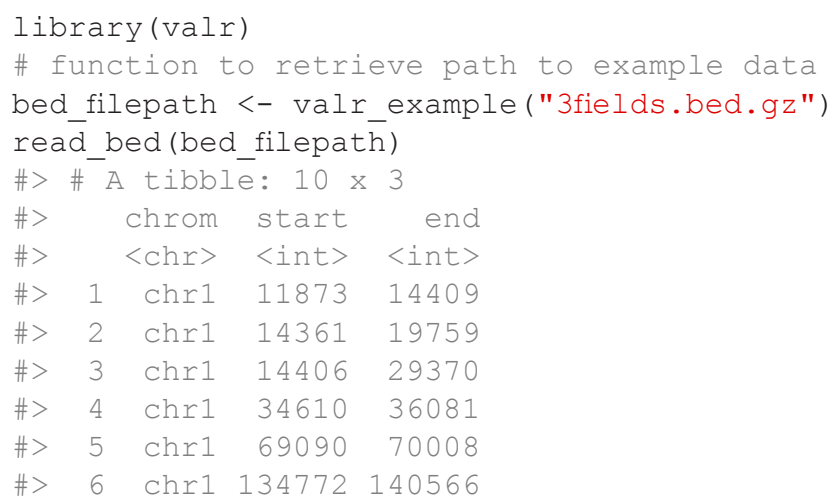




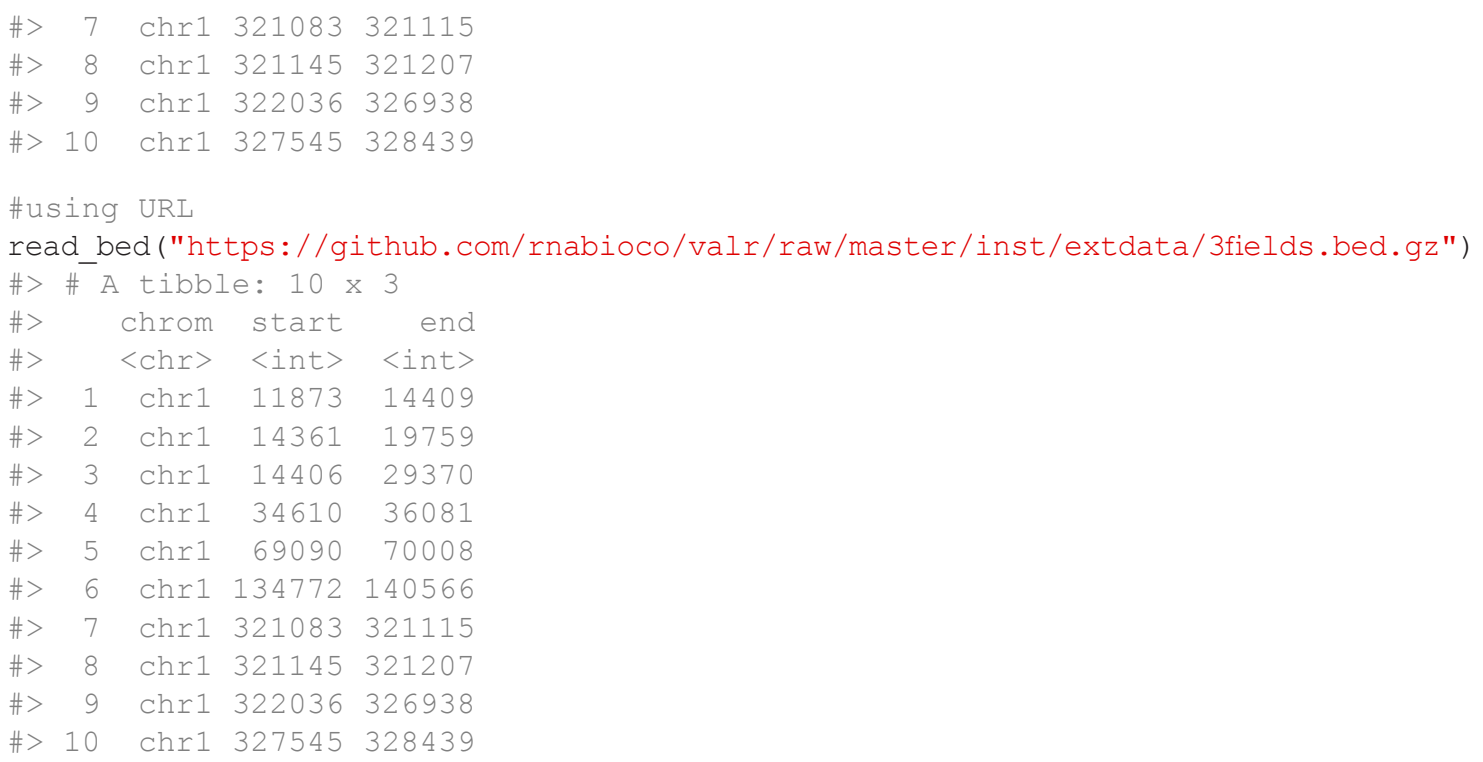

Example of combining valr tools. The functions in valr have similar names to their BEDtools counterparts, and so will be familiar to users of the BEDtools suite. Also, similar to pybedtools ${ }^{10}$, a python wrapper for BEDtools, valr has a terse syntax. For example, shown below is a demonstration of how to find all intergenic SNPs within 1 kilobase of genes using valr. The BED files used in the following examples are described in the Data Availability section.

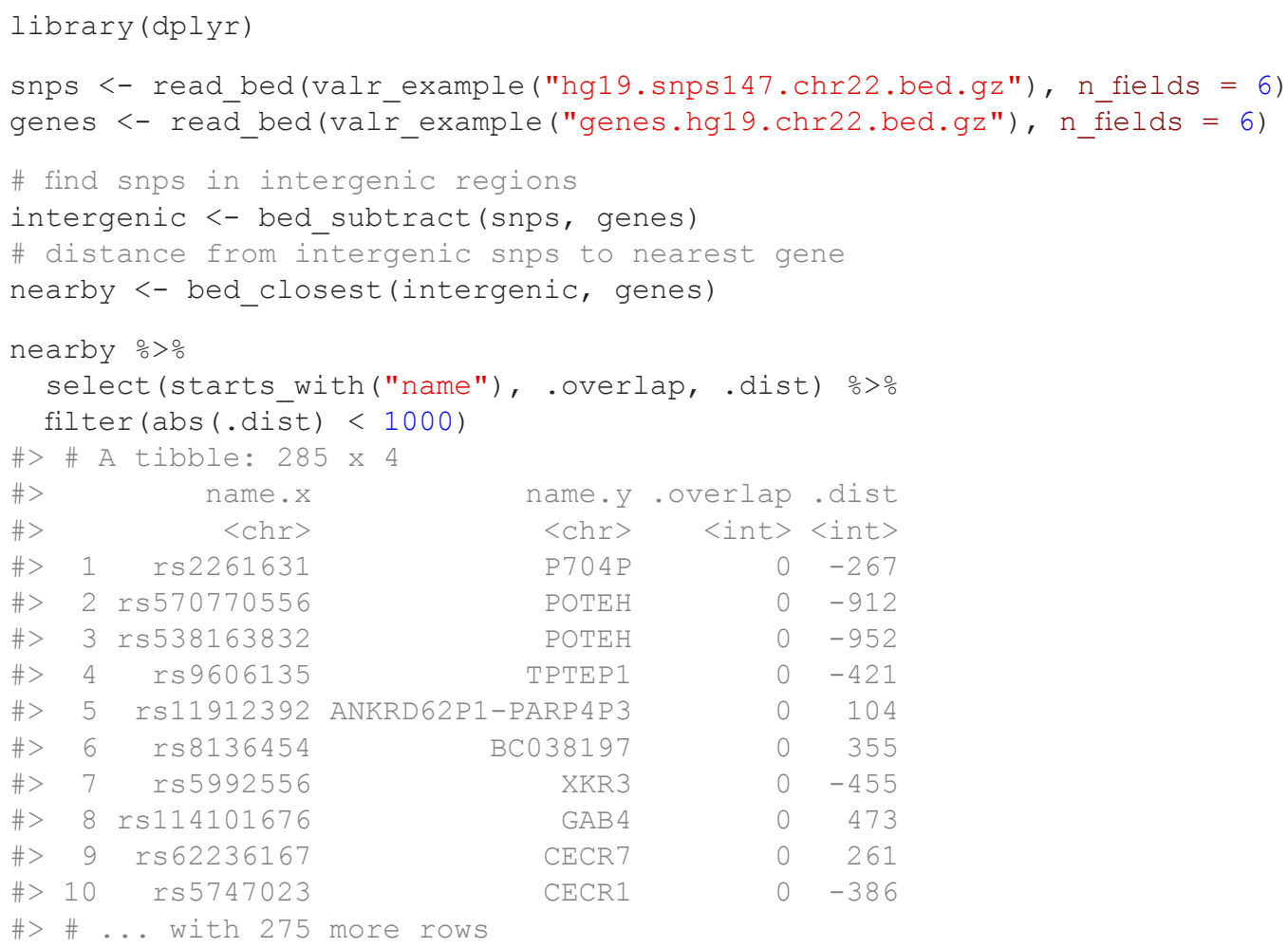

Visual documentation. By conducting interval arithmetic entirely in $\mathrm{R}$, val $\mathrm{r}$ is also an effective teaching tool for introducing interval analysis to early-stage analysts without requiring familiarity with both command-line tools and R. To aid in demonstrating the interval operations available in valr, we developed the bed glyph () tool which produces plots demonstrating the input and output of operations in valr in a manner similar to those found in the BEDtools documentation. Shown below is the code required to produce glyphs displaying the results of intersecting $\mathrm{x}$ and $\mathrm{y}$ intervals with bed_intersect (), and the result of merging $\mathrm{x}$ intervals with bed_merge () (Figure 1). 

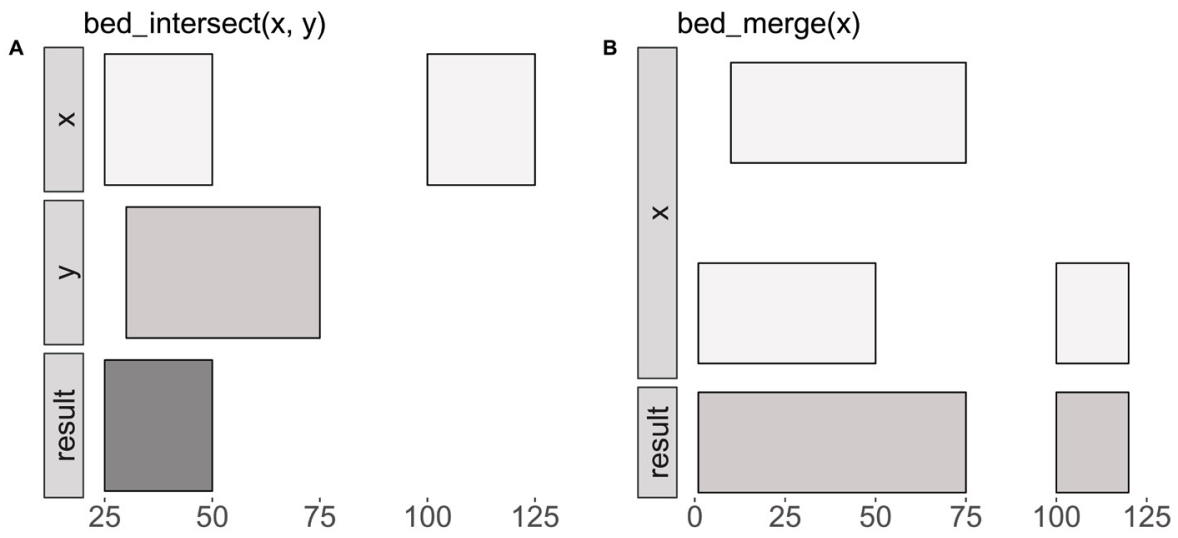

Figure 1. Visualizing interval operations in valr with bed_glyph().

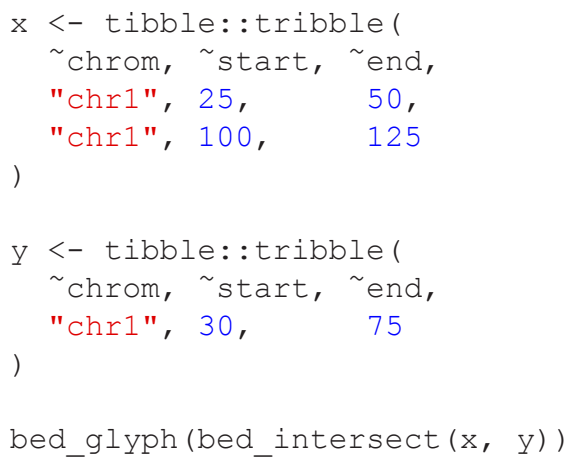

And this glyph illustrates bed merge ( ) :

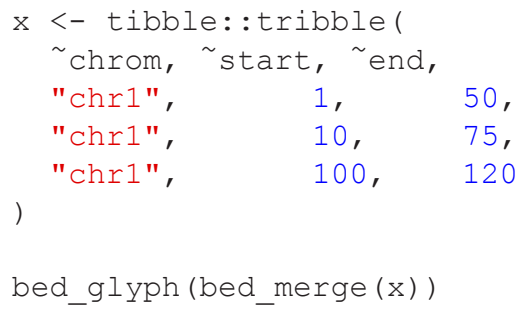

Grouping data. The group by function in dplyr can be used to execute functions on subsets of single and multiple data_frames. Functions in valr leverage grouping to enable a variety of comparisons. For example, intervals can be grouped by strand to perform comparisons among intervals on the same strand.

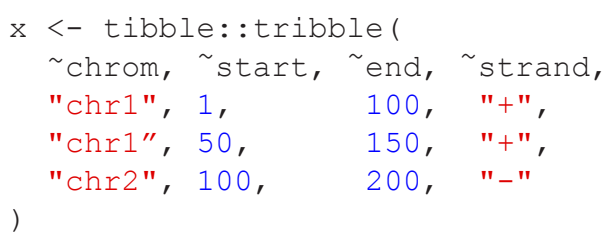




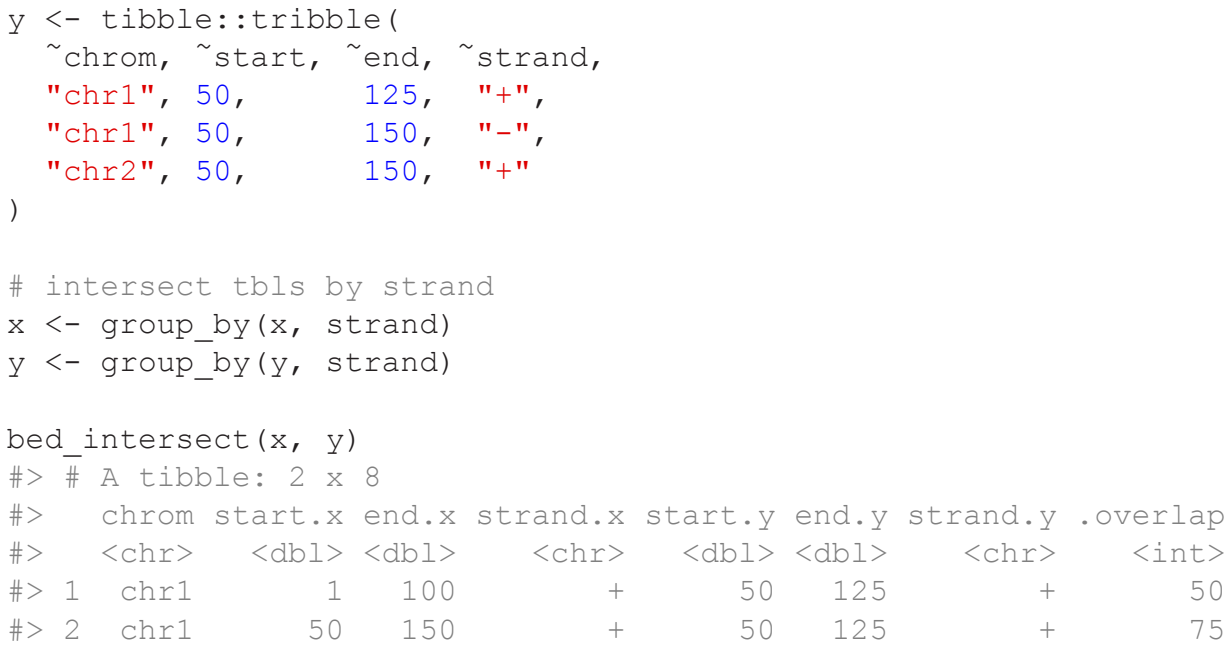

Comparisons between intervals on opposite strands are done using the flip_strands () function:

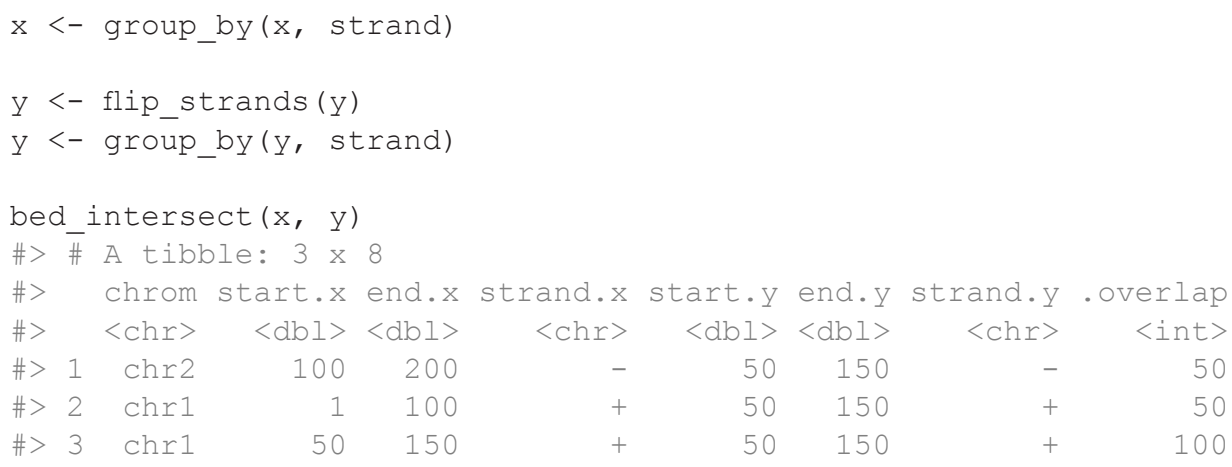

Both single set (e.g. bed_merge ( ) ) and multi set operations will respect groupings in the input intervals.

Column specification. Columns in BEDtools are referred to by position:

\# calculate the mean of column 6 for intervals in 'b' that overlap with 'a' bedtools map - a a.bed -b b.bed -c 6 -o mean

In $\mathrm{valr}$, columns are referred to by name and can be used in multiple name/value expressions for summaries.

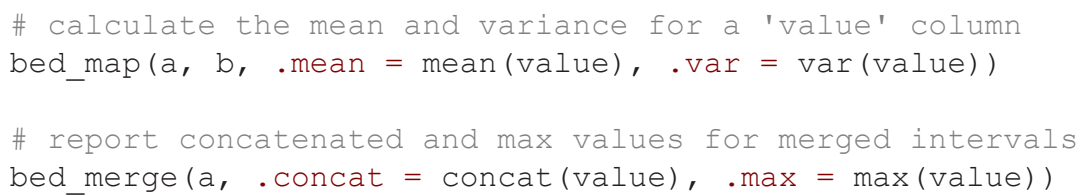

$\boldsymbol{A P I}$. The major functions available in valr are shown in Table 1. 


\begin{tabular}{|c|c|}
\hline Function Name & Purpose \\
\hline \multicolumn{2}{|l|}{ Reading Data } \\
\hline read_bed & Read BED files \\
\hline read_bedgraph & Read bedGraph files \\
\hline read_narrowpeak & Read narrowPeak files \\
\hline read_broadpeak & Read broadPeak files \\
\hline \multicolumn{2}{|l|}{ Interval Transformation } \\
\hline bed_slop & Expand interval coordinates \\
\hline bed_shift & Shift interval coordinates \\
\hline bed_flank & Create flanking intervals \\
\hline bed_merge & Merge overlapping intervals \\
\hline bed_cluster & Identify (but not merge) overlapping intervals \\
\hline bed_complement & Create intervals not covered by a query \\
\hline \multicolumn{2}{|l|}{ Interval Comparison } \\
\hline bed_intersect & Report intersecting intervals from $x$ and $y$ tbls \\
\hline bed_map & Apply functions to selected columns for overlapping intervals \\
\hline bed_subtract & Remove intervals based on overlaps \\
\hline bed_window & Find overlapping intervals within a window \\
\hline bed_closest & Find the closest intervals independent of overlaps \\
\hline \multicolumn{2}{|l|}{ Randomizing intervals } \\
\hline bed_random & Generate random intervals from an input genome \\
\hline bed_shuffle & Shuffle the coordinates of input intervals \\
\hline \multicolumn{2}{|l|}{ Interval statistics } \\
\hline $\begin{array}{l}\text { bed_fisher, bed_ } \\
\text { projection }\end{array}$ & $\begin{array}{r}\text { Calculate significance of overlaps between two sets of } \\
\text { intervals }\end{array}$ \\
\hline bed_reldist & Quantify relative distances between sets of intervals \\
\hline bed_absdist & Quantify absolute distances between sets of intervals \\
\hline bed_jaccard & Quantify extent of overlap between two sets of intervals \\
\hline \multicolumn{2}{|l|}{ Utilities } \\
\hline bed_glyph & Visualize the actions of valr functions \\
\hline bound_intervals & Constrain intervals to a genome reference \\
\hline bed_makewindows & Subdivide intervals \\
\hline bed12_to_exons & Convert BED12 to BED6 format \\
\hline interval_spacing & Calculate spacing between intervals \\
\hline db_ucsc, db_ensembl & Access remote databases \\
\hline
\end{tabular}

Summarizing interval coverage across genomic features

This demonstration illustrates how to use valr tools to perform a "meta-analysis" of signals relative to genomic features. Here we analyze the distribution of histone marks surrounding transcription start sites, using H3K4Me3 Chip-Seq data from the ENCODE project.

First we load packages and relevant data.

bedfile <- valr example("genes.hg19.chr22.bed.gz")

genomefile <- valr_example("hg19.chrom.sizes.gz")

bgfile <- valr_example("hela.h3k4.chip.bg.gz") 


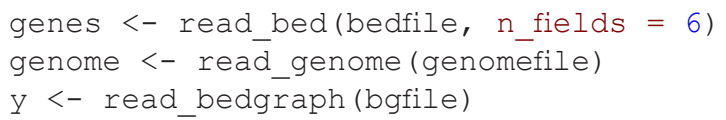

Then, we generate $1 \mathrm{bp}$ intervals to represent transcription start sites (TSSs). We focus on + strand genes, but - genes are easily accommodated by filtering them and using bed_makewindows () with reversed window numbers.

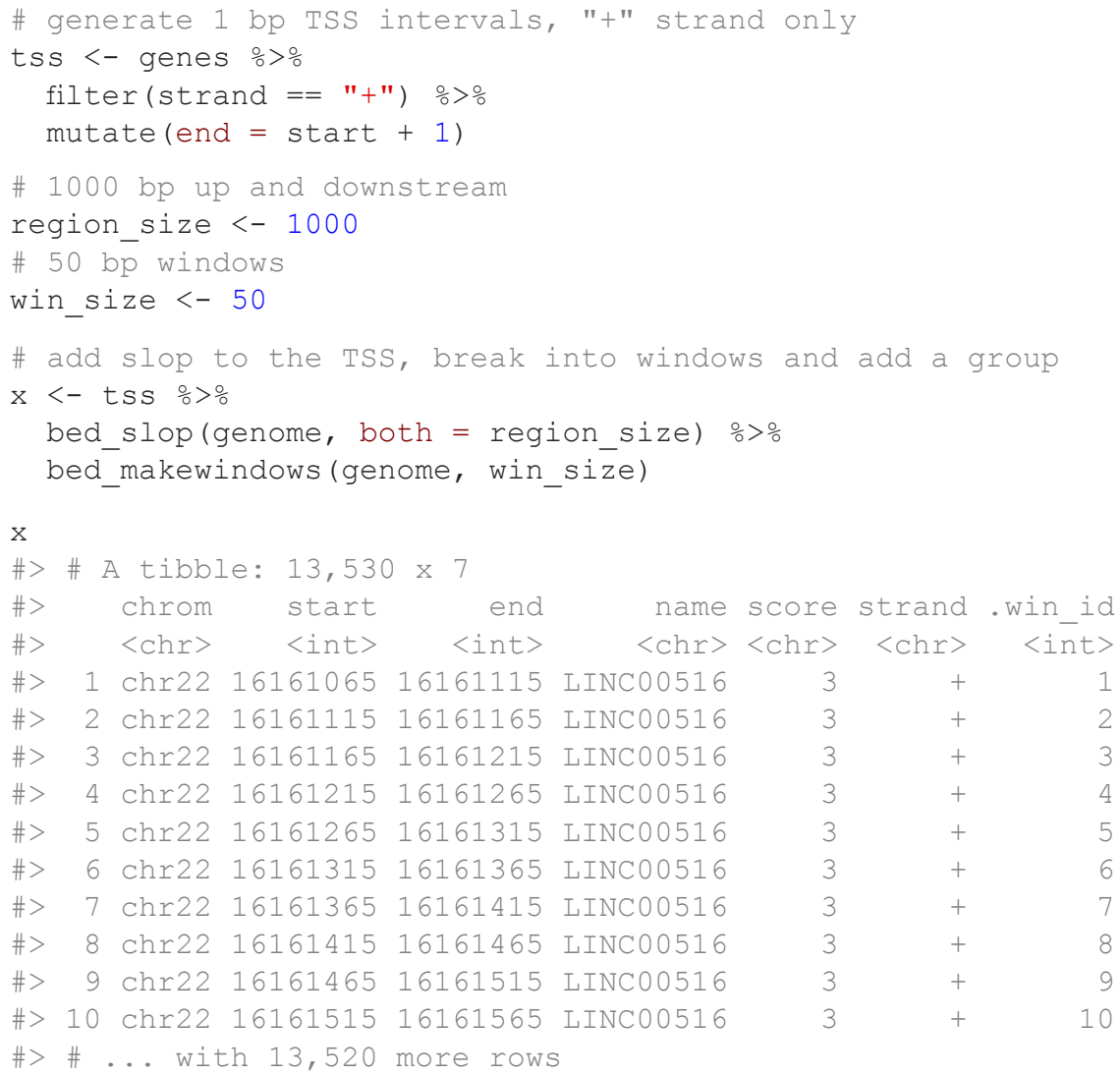

Now we use the .win id group with bed map ( ) to calculate a sum by mapping y signals onto the intervals in $\mathrm{x}$. These data are regrouped by .win id and a summary with mean and sd values is calculated.

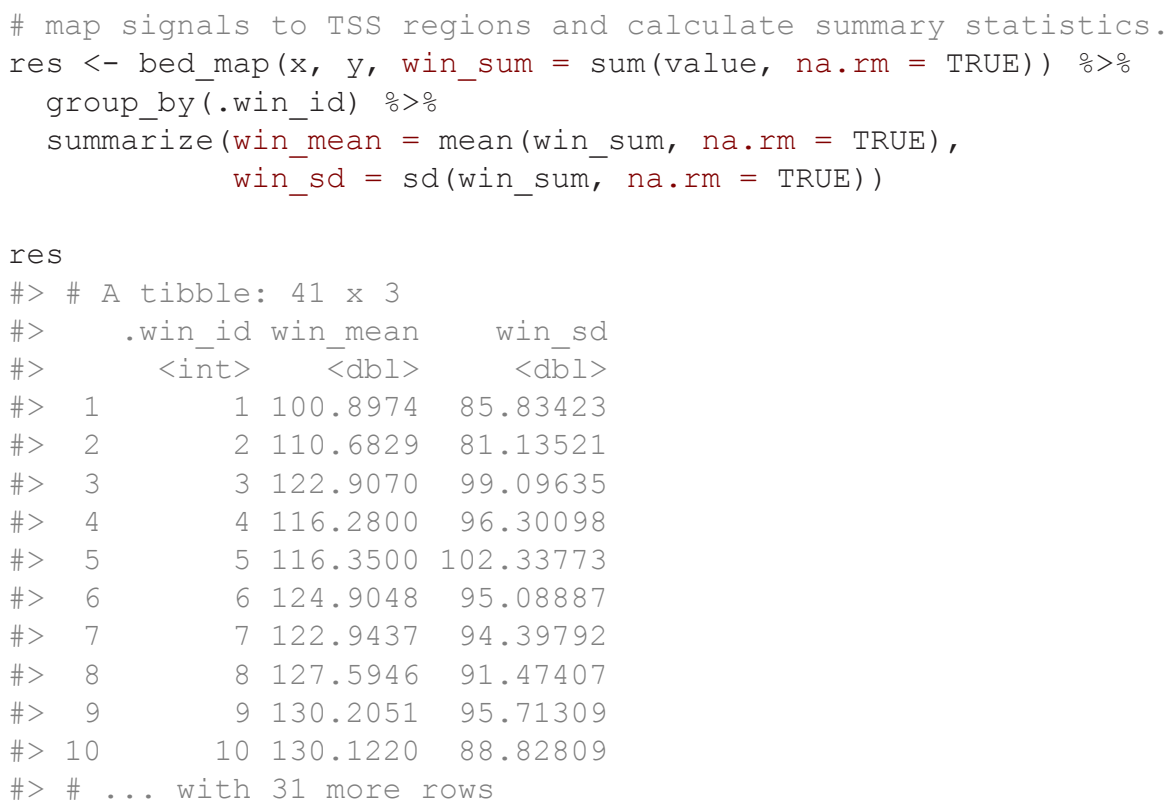

Finally, these summary statistics are used to construct a plot that illustrates histone density surrounding TSSs (Figure 2). 


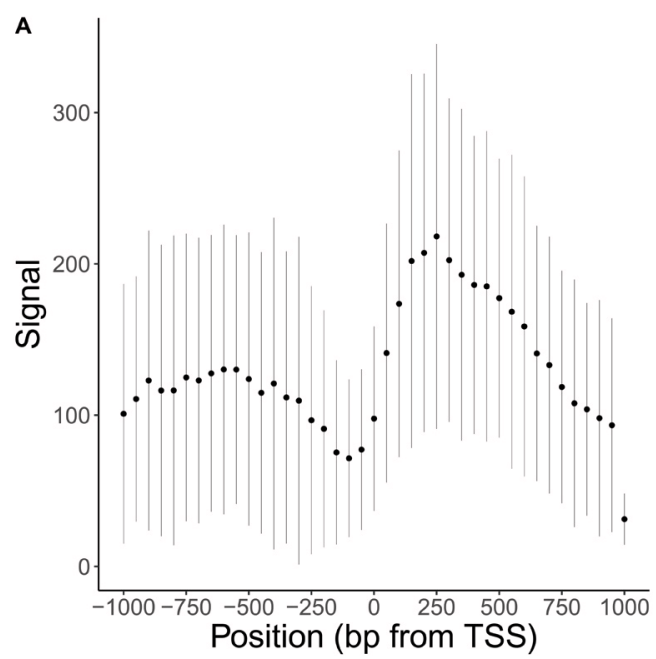

Figure 2. Meta-analysis of signals relative to genomic features with valr. (A) Summarized coverage of human H3K4Me3 Chip-Seq coverage across positive strand transcription start sites on chromosome 22. Data presented +/- SD.

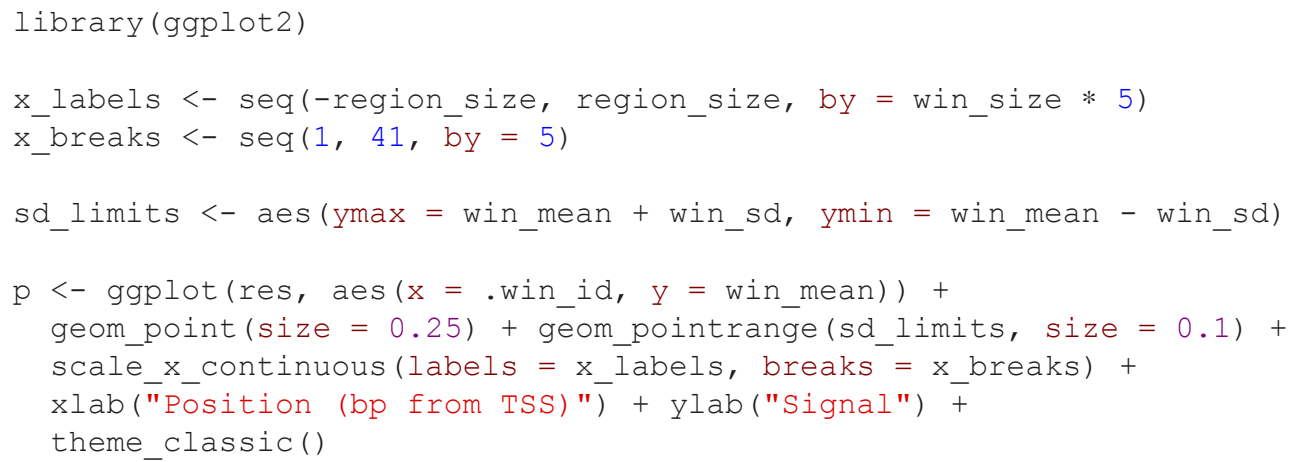

Interval statistics

Estimates of significance for interval overlaps can be obtained by combining bed_shuffle (), bed_random () and the sample_functions from dplyr with interval statistics in valr.

Here, we examine the extent of overlap of repeat classes (repeatmasker track obtained from the UCSC genome browser) with exons in the human genome (hg19 build, on chr22 only, for simplicity) using the jaccard similarity index. bed jaccard() implements the jaccard test to examine the similarity between two sets of genomic intervals. Using bed_shuffle() and replicate() we generate a data frame containing 100 sets of randomly selected intervals then calculate the jaccard index for each set against the repeat intervals to generate a null-distribution of jaccard scores. Finally, an empirical p-value is calculated from the null-distribution.

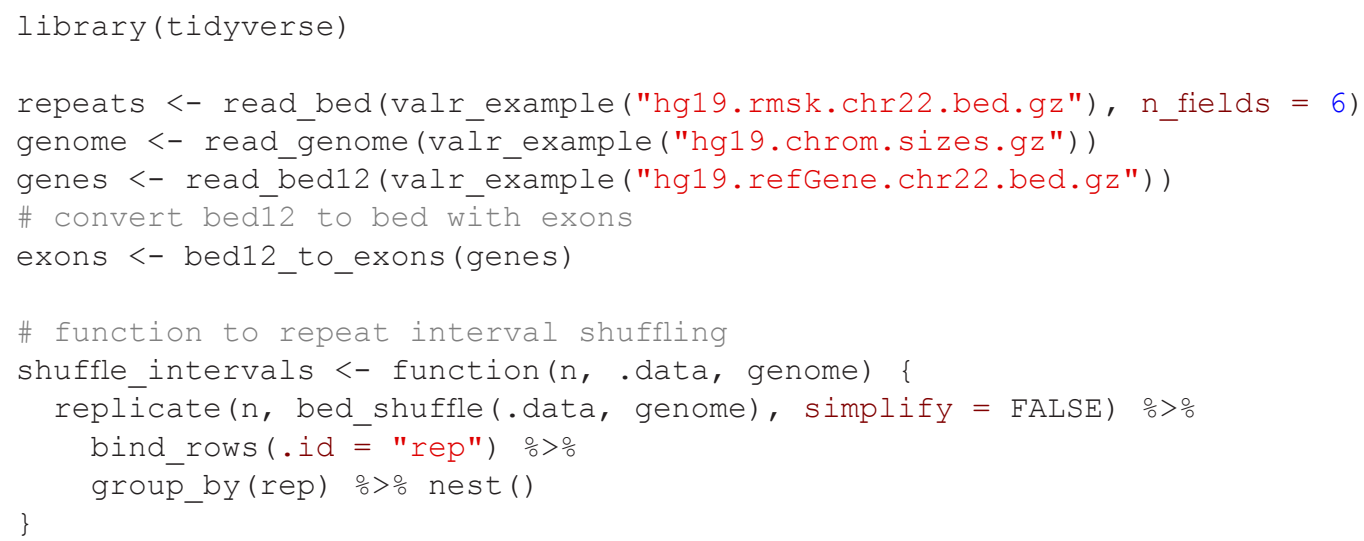




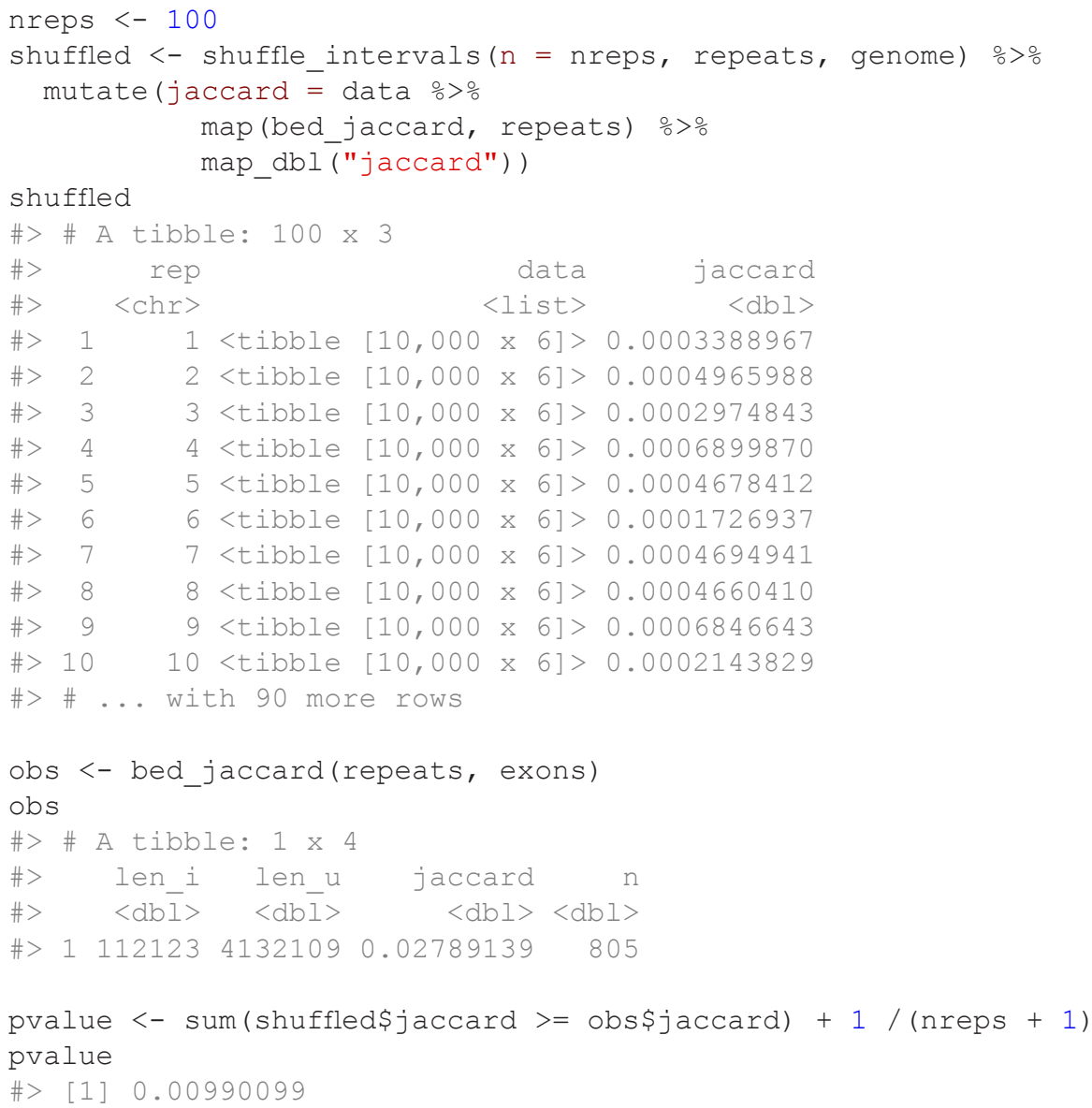

Benchmarking against bedtools

In order to ensure that valr performs fast enough to enable interactive analysis, key functionality is implemented in $\mathrm{C}++$. To test the speed of major valr functions we generated two data frames containing 1 million randomly selected 1 kilobase intervals derived from the human genome (hg19). Most of the major valr functions complete execution in less than 1 second, demonstrating that valr can process large interval datasets efficiently (Figure $3 \mathrm{~A}$ ).

We also benchmarked major valr functions against corresponding commands in BEDtools. valr operates on data_frames already loaded into RAM, whereas BEDtools performs file-reading, processing, and writing. To compare valr against BEDtools we generated two BED files containing 1 million randomly selected 1 kilobase intervals derived from the human genome (hg19). For valr functions, we timed reading the table into R (e.g. with read bed ( ) ) and performing the respective function. For BEDtools commands we timed executing the command with the output written to / dev/null. valr functions performed similarly or faster than BEDtools commands, with the exception of bed_map and bed_fisher (Figure 3B).

Reproducible reports and interactive visualizations

Command-line tools like BEDtools and bedops can be incorporated into reproducible workflows (e.g., with snakemake ${ }^{11}$ ), but it is cumbersome to transition from command-line tools to exploratory analysis and plotting software. RMarkdown documents are plain text files, amenable to version control, which provide an interface to generate feature rich PDF and HTML reports that combine text, executable code, and figures in a single document. valr can be used in RMarkdown documents to provide rapid documentation of exploratory data analyses and generate reproducible work-flows for data processing. Moreover, new features in RStudio, such as notebook viewing, and multiple language support enable similar functionality to another popular notebook platform jupyter notebooks. 
A

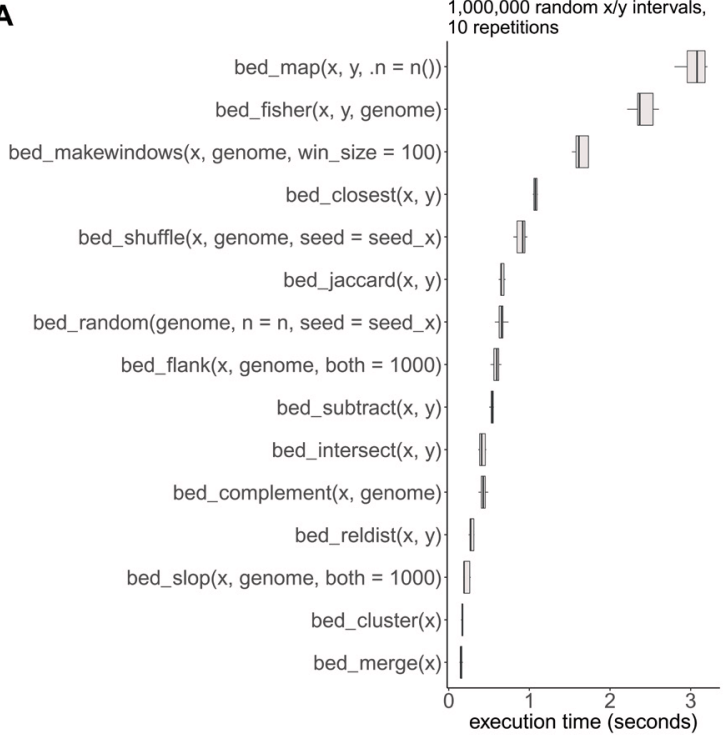

B

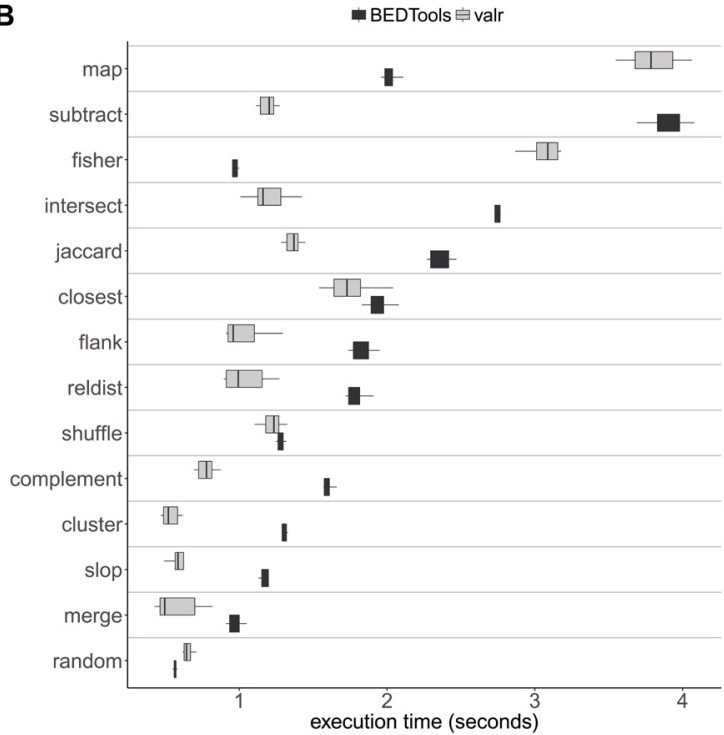

Figure 3. Performance of val $\mathrm{r}$ functions. (A) Timings were calculated by performing 10 repetitions of indicated functions on data frames preloaded in $\mathrm{R}$ containing 1 million random 1 kilobase $x / y$ intervals generated using bed_random (). (B) Timings for executing functions in BEDtools v2.25.0 or equivalent functions in valr using the same interval sets as in (A) written to files. All BEDtools function outputs were written to / dev/null/, and were timed using GNU time. Timings for valr functions in (B) include times for reading files using read bed () functions and were timed using the microbenchmark package.

Additionally, valr seamlessly integrates into R shiny $\mathrm{y}^{12}$ applications allowing for complex interactive visualizations relating to genomic interval analyses. We have developed a shiny application (available on Gitu.b) that explores ChiP-Seq signal density surrounding transcription start sites and demonstrates the ease of implementing valr to power dynamic visualizations.

\section{Summary}

valr provides a flexible framework for interval arithmetic in R/Rstudio. valr functions are written with a simple and terse syntax that promotes flexible interactive analysis. Additionally by providing an easy-to-use interface for interval arithmetic in R, valr is also a useful teaching tool to introduce the analyses necessary to investigate correlations between genomic intervals, without requiring familiarity with the command-line. We envision that valr will help researchers quickly and reproducibly analyze genome interval datasets.

\section{Data and software availability}

The valr package includes external datasets stored in the inst/extdata/ directory that were used in this manuscript. These datasets were obtained from the ENCODE Project ${ }^{13}$ or the UCSC genome browser ${ }^{14}$. BED files were generated by converting the UCSC tables into BED format. BED and BEDgraph data was only kept from chromosome 22, and was subsampled to produce file sizes suitable for submission to the CRAN repository. The original raw data is available from the following sources:

hela.h3k4.chip.bg.gz SRA record: SRR227441, ENCODE identifier: ENCSR000AOF

hg19.refGene.chr22.bed.gz ftp://hgdownload.soe.ucsc.edu/goldenPath/hg19/database/ refGene.txt.gz

hg19.rmsk.chr22.bed.gz ftp://hgdownload.soe.ucsc.edu/goldenPath/hg19/database/rmsk. txt.gz

hg19.chrom.sizes.gz ftp://hgdownload. soe.ucsc.edu/goldenPath/hgl9/database/ chrominfo.txt.gz

genes.hg19.chr22.bed.gz ftp: //hgdownload.soe.ucsc.edu/goldenPath/hg19/database/refGene.txt.gz

hg19.snps147.chr22.bed.gz ftp://hgdownload.soe.ucsc.edu/goldenPath/hg19/database/ snp147.txt.gz 
valr can be installed via CRAN using install.packages ("valr").

valr is maintained at http://github.com/rnabioco/valr.

Latest valr source code is available at http://github.com/rnabioco/valr.

The latest stable version of source code is at: https://github.com/rnabioco/valr/archive/ v0.3.0.tar.gz

Archived source code at the time of publication: http://doi org/10.5281/ zenodo.81540 $03^{15}$

License: MIT license.

Competing interests

No competing interests were disclosed.

Grant information

This work was supported by the RNA Bioscience Initiative (funded by a Transformational Research Award from the University of Colorado School of Medicine), a grant from the National Institutes of Health (R35 GM119550 to J.H.), the Colorado Office of Economic Development and International Trade (CTGGI 2016-2096), the BioFrontiers Computing Core at the BioFrontiers Institute, University of Colorado at Boulder and the Intramural Research Program of the National Library of Medicine.

The funders had no role in study design, data collection and analysis, decision to publish, or preparation of the manuscript.

\section{Acknowledgments}

This work was in part completed during an NIH sponsored Hackathon hosted by the Biofrontiers Department at the University of Colorado at Boulder.

1. Quinlan AR, Hall IM: BEDTools: a flexible suite of utilities for comparing genomic features. Bioinformatics. 2010; 26(6): 841-842. PubMed Abstract | Publisher Full Text | Free Full Text

2. Neph S, Kuehn MS, Reynolds AP, et al:: BEDOPS: highperformance genomic feature operations. Bioinformatics. 2012; 28(14): 1919-1920.

PubMed Abstract | Publisher Full Text | Free Full Text

3. Lawrence $M$, Huber $W$, Pagès $\mathrm{H}$, et al.: Software for computing and annotating genomic ranges. PLoS Comput Biol. 2013; 9(8): e1003118.

PubMed Abstract | Publisher Full Text | Free Full Text

4. Haider $\mathrm{S}$, Waggott $\mathrm{D}$, Lalonde $\mathrm{E}$, et al:: A bedr way of genomic interval processing. Source Code Biol Med. 2016; 11: 14. PubMed Abstract | Publisher Full Text | Free Full Text

5. Favorov A, Mularoni L, Cope LM, et al.: Exploring massive, genome scale datasets with the GenometriCorr package. PLoS Comput Biol. 2012; 8(5): e1002529.

PubMed Abstract | Publisher Full Text | Free Full Text

6. Wickham H: tidyverse: Easily Install and Load 'Tidyverse' Packages. $\mathrm{R}$ package version 1.1.1, 2017. Reference Source

7. Wickham H, Francois R: dplyr: A Grammar of Data Manipulation $R$ package version $0.5 .0,2016$

Reference Source

8. Eddelbuettel D, François R: Rcpp: Seamless R and $\mathbf{C + +}$ integration.
Stat Softw 2011; 40(8): 1-18

Publisher Full Text

9. Cormen $\mathrm{TH}$, Leiserson CE, Rivest RL, et al.: Introduction to Algorithms. 2nd Ed. Cambridge (Massachusetts): MIT Press; 2001. Reference Source

10. Dale RK, Pedersen BS, Quinlan AR: Pybedtools: a flexible Python library for manipulating genomic datasets and annotations. Bioinformatics. 2011; 27(24): 3423-3424. PubMed Abstract | Publisher Full Text | Free Full Text

11. Köster J, Rahmann S: Snakemake--a scalable bioinformatics workflow engine. Bioinformatics 2012; 28(19): 2520-2522. PubMed Abstract | Publisher Full Text

12. Chang W, Cheng J, Allaire JJ, et al:: shiny: Web Application Framework for R. R package version 1.0.3. 2017. Reference Source

13. ENCODE Project Consortium: An integrated encyclopedia of DNA elements in the human genome. Nature. 2012; 489(7414): 57-74. PubMed Abstract | Publisher Full Text | Free Full Text

14. Rosenbloom KR, Armstrong J, Barber GP, et al:: The UCSC Genome Browser database: 2015 update. Nucleic Acids Res. 2015; 43(Database issue): D670-D681. PubMed Abstract | Publisher Full Text | Free Full Text

15. Hesselberth J, kriemo, sheridar, et al.: rnabioco/valr: Zenodo release. Zenodo. 2017.

Data Source 


\section{Open Peer Review}

\section{Current Peer Review Status:}

\section{Version 1}

Reviewer Report 10 July 2017

https://doi.org/10.5256/f1000research.12975.r24101

(C) 2017 Dale R. This is an open access peer review report distributed under the terms of the Creative Commons Attribution License, which permits unrestricted use, distribution, and reproduction in any medium, provided the original work is properly cited.

\section{Ryan K. Dale}

Laboratory of Cellular and Developmental Biology, National Institute of Diabetes and Digestive and Kidney Diseases, National Institutes of Health, Bethesda, MD, USA

The authors describe a package for manipulating genomic interval data in $\mathrm{R}$ using principles from the "tidyverse" for the data structures and API. This sets it apart from existing tools such as GenomicRanges or bedr which have their own ways of storing and manipulating data. As a result, valr should be easier to pick up and integrate with the rest of the R ecosystem, and the "tidyverse" in particular. Illustrative examples give the reader a taste for the package while highlighting the novel features.

In general, this looks to be a very useful tool. The code quality is excellent and it is great to see so many tests including the addition of regression tests as issues are identified.

My comments are very minor:

Group-by code listing: comment "\# intersect tbls by strand" should be "\# group tbls by strand"

Bioconductor might be a more appropriate repository than CRAN

Description of in-memory usage: I see from the software documentation that BAM and VCF will be supported in the future, and the documentation explicitly mentions that valr operates on data in-memory. The section comparing with BEDTools briefly mentions the inmemory aspect, but it would be helpful to be clearer about memory usage in the manuscript, especially as users attempting to use large BAM files may run out of memory.

This is just a suggestion for improvement: Over the years, numerous bugs from corner cases have been found and handled in BEDTools. It would greatly increase confidence in the underlying algorithms you have written if there is input/output parity between valr and BEDTools, at least for the tools that overlap the two packages. For example I see some test 
cases that use input from the BEDTools test suite (e.g., test_cluster.r), but don't check the output. It should be straightforward to check the output against that provided by the BEDTools test suite. Correspondingly it would be good for BEDTools to use valr input/expected output in its test suite.

Is the rationale for developing the new software tool clearly explained?

Yes

Is the description of the software tool technically sound?

Yes

Are sufficient details of the code, methods and analysis (if applicable) provided to allow replication of the software development and its use by others?

Yes

Is sufficient information provided to allow interpretation of the expected output datasets and any results generated using the tool?

Yes

Are the conclusions about the tool and its performance adequately supported by the findings presented in the article?

Yes

Competing Interests: No competing interests were disclosed.

I confirm that I have read this submission and believe that I have an appropriate level of expertise to confirm that it is of an acceptable scientific standard.

Reviewer Report 10 July 2017

https://doi.org/10.5256/f1000research.12975.r23916

(C) 2017 Amezquita R. This is an open access peer review report distributed under the terms of the Creative Commons Attribution License, which permits unrestricted use, distribution, and reproduction in any medium, provided the original work is properly cited.

\section{Robert A. Amezquita}

Department of Immunobiology, School of Medicine, Yale University, New Haven, CT, USA

Riemondy and colleagues have made a valuable tool, 'valr', available to the general public, one which deserves much merit for bringing modern R idioms from the 'tidyverse' into the world of genomic research. As a fellow bioinformatician who has struggled with the idiosyncrasies of the aforementioned tools for interval manipulation in $\mathrm{R}$, 'valr' addresses many of the usability issues associated with these legacy methods by fundamentally altering the user experience.

Consequently, there are two main advantages to adopting 'valr' for interval manipulation in R:

ease of writing, and ease of reading, code. Thus, this referee wholeheartedly endorses 'valr', and 
hopes to see more work that brings many of the 'tidyverse' philosophies over to working with genomics in R.

Nonetheless, there is some room for improvement of the associated manuscript to better help explain the philosophy and usage of 'valr', and its place amongst the many tools for the manipulation of genomic intervals.

Firstly, in the introduction, it is mentioned that there exist 'IRanges' methods that utilize the S4 convention, whereas 'valr' utilizes a less formal schema where 3 columns, 'chrom', 'start', 'end', are present in the 'data_frame` object. Indeed, it may be of use to expand upon such design choices that were made, and what advantages/disadvantages are made in using this less formal schema, and any other highly pertinent choices that affect user experience. In addition, one line mentions integration with other 'tidyverse' tools, and should expand upon this with either one to a few specific examples or explain this point in more detail. Additionally, it should also be pointed out how 'valr` builds upon these existing toolkits, and either expands upon/adopts their conventions. One way might be to create a table comparing functions between 'valr'/bedtools/GenomicRanges might be helpful for a reader to see that the toolkit will be easily adoptable. Indeed, its mentioned that the syntax is similar to bedtools in the use cases, and might be good to mention in the introduction as well. Thus, an expanded introduction/additional section explaining the uniqueness of 'valr' would help to better "sell" when one should use 'valr' and why.

In performing benchmarking, it would be useful to include one or two leading R tools, such as GenomicRanges, into the calculations, as this is likely how many R programmers currently perform interval manipulations natively in $\mathrm{R}$, and I suspect would likely show an impressive performance improvement by relation.

'valr' presents an exciting new development in the $\mathrm{R}+\mathrm{Genomics}$ realm, and this referee is hopeful that this sort of development helps fuel further 'tidyomics' tools for $\mathrm{R}$ bound together by $\mathrm{a}$ cohesive philosophy, great user experience, and pointed utility.

Is the rationale for developing the new software tool clearly explained?

Partly

Is the description of the software tool technically sound?

Yes

Are sufficient details of the code, methods and analysis (if applicable) provided to allow replication of the software development and its use by others?

Yes

Is sufficient information provided to allow interpretation of the expected output datasets and any results generated using the tool?

Yes

Are the conclusions about the tool and its performance adequately supported by the 


\section{findings presented in the article?}

Yes

Competing Interests: No competing interests were disclosed.

Reviewer Expertise: Computational immunology

I confirm that I have read this submission and believe that I have an appropriate level of expertise to confirm that it is of an acceptable scientific standard.

The benefits of publishing with F1000Research:

- Your article is published within days, with no editorial bias

- You can publish traditional articles, null/negative results, case reports, data notes and more

- The peer review process is transparent and collaborative

- Your article is indexed in PubMed after passing peer review

- Dedicated customer support at every stage

For pre-submission enquiries, contact research@f1000.com 